\title{
Sustentabilidade e o Sistema Agropecuário de Produção Integrada ${ }^{14}$ Agricultural Sustainability and Integrated Production System

\author{
Renato Abdo ${ }^{15}$ \\ Diamantino Pereira ${ }^{16}$
}

Artigo recebido em para publicação em out/2013 e aceito para publicação em maio/2014

\begin{abstract}
RESUMO
Com base em uma pesquisa sobre as práticas agrícolas na região do Alto Tietê, particularmente em Mogi das Cruzes, Biritiba Mirim e Salesópolis, integrantes do Cinturão Verde da Região Metropolitana de São Paulo (RMSP), refletimos sobre algumas características da agricultura convencional, destacando o paradigma dominante da "Revolução Verde" lastreada na utilização de agroquímicos, sementes de alto rendimento e irrigação. Aponta as exigências dos grandes grupos varejistas para admitir e manter seus fornecedores. Relaciona esse processo com a implementação do Sistema Agropecuário de Produção Integrada (SAPI) e como as práticas ali sugeridas referentes ao controle ambiental, racionalização da aplicação de agroquímicos e controle biológico de pragas e doenças poderiam apontar para alterações nas práticas agrícolas convencionais. Refletimos ainda sobre a noção de sustentabilidade agrícola e as concepções alternativas das práticas agroecológicas e da "Revolução Duplamente Verde".
\end{abstract}

Palavras-chave: Agricultura Sustentável. Revolução Verde. Produção Integrada.

\section{ABSTRACT}

Based on a survey of agricultural practices in the Upper Tietê region, particularly in Mogi das Cruzes, Biritiba Mirim and Salesópolis, members of the Green Belt of São Paulo's Metropolitan Region, we reflect on some characteristics of conventional agriculture, highlighting the dominant paradigm of the "Green Revolution" backed the use of pesticides, high-yield seeds and irrigation. Pointing the requirements of large retail groups to hire and keep their suppliers. This process relates to the implementation of the Agricultural System of Integrated Production and how the practices suggested here regarding environmental control, streamlining the application of pesticides and biological control of pests and diseases could point to changes in conventional agricultural practices. Still reflect on the concept of agricultural sustainability and alternative conceptions of agroecological practices and "Doubly Green Revolution".

Keywords: Sustainable Agriculture. Green Revolution. Integrated Production.

\section{INTRODUÇÃO}

O presente artigo baseou-se em projeto de pesquisa que contemplou ações relativas à caracterização do processo de trabalho e produção em propriedades

14 Este trabalho relaciona-se com projeto de pesquisa financiado pela Fundação de Amparo à Pesquisa do Estado de São Paulo - FAPESP

${ }^{15}$ Graduação em Engenharia Agronômica pela Universidade de Taubaté (UNITAU), Especialização em Gestão e Manejo Ambiental em Sistemas Agrícolas pela Universidade Federal de Lavras (UFLA) e Mestrado pelo Programa de Mudança Social e Participação Política da Escola de Artes, Ciências e Humanidades da Universidade de São Paulo (EACH-USP). Coordenador de Agronegócios do Sindicato Rural de Mogi das Cruzes. E-mail: renato.abdo@ig.com.br

${ }_{16}$ Graduação e Mestrado em Geografia pela Universidade de São Paulo (USP), Doutorado em Sociologia pela Pontifícia Universidade Católica de São Paulo (PUCSP), Professor da Escola de Artes, Ciências e Humanidades da Universidade de São Paulo (EACH-USP). E-mail: diamantino@usp.br 
agrícolas de olericultura, detalhando a utilização de tecnologia e a estruturação territorial da propriedade. Particularmente importante foi o monitoramento dos procedimentos produtivos (gestão do trabalho, da área de produção, de resíduos e poluentes, aplicação de fertilizantes e defensivos, irrigação, colheita e processamento) em uma grande propriedade. A partir dos elementos trazidos à tona nesse processo pudemos expandir a caracterização do conjunto dos procedimentos para grande parte das unidades de produção da região contando com o auxílio do Sindicato Rural de Mogi das Cruzes.

Essa caracterização nos possibilitou destacar os principais elementos constituintes da condução do processo produtivo e refletir sobre eles. Identificamos então o conteúdo da política do "Sistema Agropecuário de Produção Integrada" e refletimos sobre as contingências de sua implantação nas unidades de produção de hortaliças, as necessidades de investimentos, adequação gerencial e treinamento de mão de obra que se fariam necessárias, colocadas ao lado dos possíveis benefícios em termos de rentabilidade e sustentabilidade da produção.

\section{AGRICULTURA E SUSTENTABILIDADE}

A agricultura denominada de "subsistência" na qual se produzia para consumo próprio além de excedentes para troca ou comercialização há muito tempo não é o processo predominante das atividades agrícolas. Atualmente, mesmo as pequenas propriedades produzem essencialmente para o mercado e dessa forma buscam conseguir os recursos com os quais compram os bens necessários para sua manutenção. Ou seja, as novas formas de organização do trabalho e da produção constroem uma nova dimensão espacial, sem provocar o desaparecimento em muitos lugares de retratos antigos, gestados em outra dinâmica social. (Pereira, 2001, p.45)

Há tempos, como veremos nas próximas páginas, a artificialização dos procedimentos agropecuários tornou-se padrão e questões como a fertilidade do solo e combate a pragas e doenças das plantas passaram a ser predominantemente enfrentados com métodos de combate químicos.

Com a hegemonia desses procedimentos agroquímicos, a antiga agricultura baseada em métodos naturais e orgânicos foi caindo no esquecimento e progressivamente os "agricultores literalmente desaprenderam essas práticas" 
(Khatounian, 2001, p. 45) colocando o seu resgate na atualidade um desafio para a implementação do desenvolvimento sustentável da agricultura.

Abordar a questão do desenvolvimento sustentável ou da sustentabilidade é sempre uma tarefa complexa na medida em que os termos apresentam significados variados dependendo de quem os utiliza. Entretanto é muito comum apresentar o conceito consagrado no relatório Brundtland para considerar que estaria tudo resolvido e, assim, esses conceitos estariam relacionados com a satisfação das "necessidades presentes, sem comprometer a capacidade das gerações futuras de suprir suas próprias necessidades." (Brundtland, 1988, p.9).

Acontece que a afirmação é apenas uma declaração de princípio e, mesmo entre os que concordam com o seu conteúdo, as interpretações e derivações são extremamente diversificadas, pois se trata efetivamente de identificar os elementos centrais que possibilitem a consecução dos objetivos apontados.

Então as dimensões ambientais propriamente ditas vêm à tona quando se colocam em tela a conservação e/ou racionalização da exploração e uso dos recursos naturais. Mas isso não basta, pois logo pode se afirmar com razão que esses processos ambientais não podem ser enquadrados sem a vinculação aos fatores sociais e econômicos. Temos então a dimensão socioambiental. (Masera, 2000, p. 12 e 13)

Como a miríade de concepções é quase infinita, alguns autores buscam identificar os pontos em que a maioria concorda, como é o caso de Gliessman (2005, p. 52) que afirma existir em relação à sustentabilidade uma "concordância geral de que ela tem uma base ecológica". Perspectiva diferenciada é apontada por Altieri quando afirma que "os enfoques que percebem o problema da sustentabilidade somente como um desafio tecnológico da produção não conseguem chegar às razões fundamentais da não sustentabilidade dos sistemas agrícolas." (Altieri, 2004, p.21)

Apesar de considerar o conceito de sustentabilidade controverso, Altieri o pondera que sua aplicação na agricultura seria útil pois isso implicaria que se "reconhece que a agricultura é afetada pela evolução dos sistemas socioeconômicos e naturais". (Altieri, op. cit. p. 20)

Claro, a discussão da sustentabilidade é dirigida à sociedade atual como um todo e, quando foi sistematizada no relatório Brundtland, o foco principal dirigia-se 
sobretudo ao desenvolvimento industrial e urbano, mas é inegável que a agricultura também apresenta responsabilidade sobre o problema da degradação ambiental (Conway, 2003, p. 194)

E essa responsabilidade coloca-se especialmente quando se quase universaliza a denominada agricultura convencional caracterizada pela extensão das monoculturas, adoção a mecanização e administração de agroquímicos.

Principalmente após a II Guerra Mundial, o arsenal agroquímico tinha se multiplicado para atuação em praticamente todos os processos agrícolas, numa profusão de adubos, inseticidas, fungicidas e herbicidas.

Assistimos então ao desenvolvimento de variedades de cultivares de alto rendimento que, entretanto, só se tornava alto, na medida em que utilizavam intensivamente o arsenal químico e dispunha de fornecimento de água constante e em abundância. Tratava-se do famoso "pacote" da Revolução Verde que se torna uma espécie de padrão de agricultura moderna e eficiente e com esse rótulo se propaga por todo o mundo e se torna o tipo de agricultura que hoje denominamos de convencional.

Essa forma de conduzir a produção agrícola artificializa os ciclos produtivos e faz com que seja considerado normal o oferecimento dos mesmos produtos agrícolas durante todo $\mathrm{o}$ ano, desconsiderando os períodos propícios para 0 desenvolvimento de cada cultura em particular.

A entrada progressiva dos supermercados na comercialização das hortaliças, fato que anteriormente era quase uma exclusividade das feiras livres, acabou por estabelecer um novo tipo de relação com os produtores agrícolas: os produtos devem ser apresentados em um formato padrão e ter peso determinado, mesmo quando a comercialização não é feita por peso e sim por unidade.

Enfim, a viabilização comercial do produtor agrícola é colocada em questão e isso se torna mais um elemento de pressão para a adoção das práticas convencionais. O outro elemento de pressão está relacionado com os custos de produção, particularmente os custos da mão de obra que é mais exigida quando não se lida com os agroquímicos. 


\section{PRODUÇÃO, TRABALHO E TÉCNICA}

A manutenção de um estabelecimento agrícola em atividade coloca o produtor sob o dilema de se adaptar a essas circunstâncias. A opção de manutenção da pequena propriedade com base, sobretudo no trabalho familiar permite o escape da escassez e alto custo da mão de obra, mas coloca a questão da escala da produção.

A outra opção consiste em produzir em grande escala, perseguir ganhos de produtividade, agregar de valor à produção e buscar maior controle das operações em sua totalidade, tanto no que diz respeito à produção, quanto ao seu beneficiamento e destinação.

Mesmo a opção familiar, não pode prescindir, entretanto da adoção de implementos e insumos que garantam a produtividade.

As hortaliças folhosas, principalmente a alface necessita de um fornecimento frequente e abundante de água em função da amplitude de sua área foliar e das características de seu sistema radicular.

A disponibilidade de água coloca-se então como uma questão estratégica. Mesmo em regiões de clima úmido não observamos a regularidade de pluviosidade necessária para o desenvolvimento pleno das plantas. Isso não ocorre na época das chuvas, e muito menos no período de estiagem, quando em algumas regiões podem transcorrer meses inteiros se uma precipitação sequer.

Se a região for bem dotada de aquíferos, com a existência de rios e córregos com grande disponibilidade de água, o problema pode ser contornado com a implantação da irrigação artificial.

A incidência de chuva direta sobre as folhosas é particularmente danosa para o seu desenvolvimento, sobretudo as chuvas intensas que são responsáveis por grandes perdas na fase de produção. Uma das alternativas para a garantia de uma produção constante sem o perigo de ocorrências dessas grandes perdas é a utilização de ambientes protegidos por telas, o que amplia a exigência de investimentos. 


\section{NUTRIÇÃO E PROTEÇÃO DAS PLANTAS}

Elementos importantes também nesse processo estão relacionados com a disponibilidade de nutrientes para o desenvolvimento adequado das plantas. $\mathrm{Na}$ agricultura convencional a adubação química é predominante. Esse fato deriva dos bons resultados alcançados em termos de produtividade com esse método e também a menor exigência de mão de obra em relação a procedimentos de adubação e compostagem orgânicos que predominavam na agricultura tradicional.

Esse tipo de adubação deveria ser precedido de análise de solo e de recomendação (receituário) agronômica, mas nem sempre ocorre dessa maneira. Pequenos e médios produtores grande parte das vezes não tem condições de contratar um agrônomo e "resolvem" essa limitação com a consulta ao agrônomo que é vinculado à revenda de fertilizantes. Evidentemente, apesar de existirem funcionários que trabalham com critério, essa combinação entre a prescrição de um produto e a sua venda normalmente não é a mais isenta possível. Fica em aberto ainda a análise do solo e a sua interpretação.

Outro elemento importante para a manutenção da produtividade é o combate às pragas e doenças. "Desde a II Guerra Mundial, a atitude comum diante de problemas com pragas, patógenos e ervas daninhas tem sido pulverizar as plantações com pesticidas." (Conway, 2003, p. 237).

Esse tipo de procedimento torna-se ainda mais caro na medida em que a aplicação é feita de maneira excessiva. Não se espera que apareça uma doença ou que ela adquira o status de dano econômico para iniciar o ataque. As pulverizações são administradas de forma preventiva, ou seja, dependendo das doenças e pragas que são previstas para aparecer em determinada época do ano e em determinadas condições climáticas.

As grandes culturas são as que apresentam os maiores índices de consumo de agroquímicos, entretanto, a prática agrícola baseada nos princípios da "revolução verde", articulando melhoria de sementes, agroquímicos e irrigação tornou-se prática rotineira da agricultura brasileira, mesmo entre os pequenos proprietários articulados que tenham alguma pretensão de colocar sua produção no mercado.

No caso das hortaliças, outra questão se coloca que é o fato da utilização de defensivos com registro para outras culturas. Em nossa pesquisa, verificamos que defensivos registrados para o cultivo do tomate, por exemplo, sendo aplicados em 
plantações de alface. A justificativa para isso é a de que as doenças e pragas já adquiriram resistência para grande parte dos defensivos registrados e os fabricantes tem se concentrado no desenvolvimento de novos produtos para as grandes culturas, sobretudo para a soja, milho, algodão e cana-de-açúcar.

Esse problema motivou inclusive um processo de regulamentação envolvendo - Mapa, a Anvisa e o lbama na formulação de um processo de regulamentação para o registro de novas formulações de agrotóxicos para as denominadas Culturas de Suporte Fitossanitário Insuficiente (CSFI), para as quais "a falta ou número reduzido de agrotóxicos e afins registrados acarreta impacto socioeconômico negativo, em função do não atendimento das demandas fitossanitárias." (Mapa/lbama/Anvisa, 2010, p. 1).

Os fabricantes concentram suas atenções nas culturas com grande demanda de agrotóxicos e para as demais, sob o argumento de que o processo de registro seria muito dispendioso, a disponibilização de novos princípios ativos e formulações têm sido muito reduzidas.

Como temos afirmado, a agricultura baseada na aplicação de agroquímicos apresenta sérios problemas. Nesse caso específico, a situação se agrava, pois nem mesmo as medidas de precaução relativas ao controle e uso dos agrotóxicos são viabilizadas pelos produtores. Sem opção efetiva no campo da agricultura convencional, os produtores agrícolas optam pela ilegalidade ao utilizar produtos formulados para outras culturas. O exemplo que demos é ilustrativo: como extrapolar a ação de um produto destinado ao tomate (fruto) para a alface, quando o que se come são as folhas, justamente as que apresentam a maior absorção dos princípios ativos?

Frente a essas questões, assim como a constatação da presença de resíduos de agrotóxicos em diversos produtos agrícolas, cresce a exigência de pelo menos um maior controle e uso racional desses produtos, quando não a sua limitação extrema.

A racionalidade que se impõe é a do controle do processo produtivo como um todo. As pressões advindas de diversos segmentos da sociedade, tanto de consumidores, quanto de órgãos ambientais e governamentais, seguramente colocarão a prática agrícola em novos patamares de produção e controle. Mesmo que não se estabeleça uma regulação ostensiva e vinculante, permanecer nessa 
atividade com condições de competitividade irá requerer articulações para além da garantia da produtividade nas propriedades.

\section{OS SUPERMERCADOS SE INSPIRAM NA REESTRUTURAÇÃO PRODUTIVA}

Uma dessas articulações vinculantes tem vindo das redes varejistas, particularmente as grandes.

$\mathrm{Na}$ atualidade, as redes varejistas passaram a estruturar seu próprio sistema de abastecimento de hortaliças, desvinculando-se das compras individualizadas em centrais de abastecimento. Querem um fornecedor que seja capaz de oferecer um mix diferenciado de produtos e que isso seja entregue higienizado e embalado todos os dias nas gôndolas. Um produtor, por mais eclético que seja, teria muitas dificuldades para articular a produção em escala de produtos diferenciados que devem constar nesse mix e então, de apenas produtores, transformaram-se também em distribuidores.

Eles recebem produtos de dezenas de produtores independentes, submetemnos à higienização e embalagem em seus galpões de processamento e fazem a entrega nos pontos de venda. Dessa maneira, eles podem fornecer todos os produtos exigidos pelo varejista sem ter que plantar todos eles. Os produtoresdistribuidores podem ainda recorrer a esses fornecedores para suprir eventuais quebras de sua própria produção.

As grandes redes credenciam os seus fornecedores através de exigências de controle do processo produtivo, da colheita e do tratamento pós-colheita. Impõem critérios mínimos de procedimentos para todas as fases da produção e fazem monitoramento em campo auditando o cumprimento dos critérios estabelecidos.

Ocorre, entretanto, que todas essas exigências das redes varejistas estão atualmente focadas apenas nas propriedades $e$ instalações do produtor credenciado, não se estendendo aos seus fornecedores.

Quando o produtor-distribuidor entrega seu produto para seus clientes, estabelece-se um sistema de responsabilidade relativa a eventuais insumos utilizados no processo produtivo que possam causar algum tipo de dano ou prejuízo aos consumidores. Note-se que, mesmo quando o produto leva a marca da rede de varejo, na maior parte das vezes ocorre a identificação do produtor na própria embalagem 
Para atender a todas essas exigências, evidentemente é necessário que o produtor apresente capacidade técnica e financeira. Técnica em relação à produção propriamente dita que deve se afastar das práticas costumeiras da agricultura convencional, mas também em relação à implantação de um sistema administrativo, sistema logístico e de controle de todos os processos. Financeira, pois se coloca a necessidade de investimentos, tanto em relação à contratação de serviços especializados, quanto à necessidade de implantar e manter a infraestrutura adequada.

\section{PRODUÇÃO INTEGRADA COMO ESTRATÉGIA COMPETITIVA?}

Definindo parâmetros de para o processo produtivo como um todo e alicerçado em um conjunto de exigências que apresenta muitos pontos em comum com as normas estabelecidas pelas grandes redes varejistas para credenciar seus fornecedores, o Ministério da Agricultura e Produção Agropecuária vem implementando há tempos a política pública de "Produção Integrada".

O conceito de Produção Integrada foi estabelecido na década de 60 do século passado pela Organização Internacional para Controle Biológico e Integrado contra os Animais e Plantas Nocivas (OILB) como um "sistema de produção que gera aumentos e demais produtos de alta qualidade, mediante a aplicação de recursos naturais e regulação de mecanismos para a substituição de insumos poluentes e a garantia da sustentabilidade da produção agrícola." (Andrigueto e Kososki, 2002, p. 7).

A produção Integrada apresenta como objetivos, entre outros, os de regular o ecossistema, preservar dos recursos naturais, minimizar os efeitos inconvenientes da atividade agrícola, assegurar o equilíbrio do ciclo dos elementos nutritivos e a fertilidade do solo. (Amaro, 2003, p.10).

Evidentemente, para que uma política se proponha a atingir todos esses objetivos, deve ser porque existe a pressuposição de eles não são observados na prática da agricultura convencional.

No Brasil, a implantação do Sistema de Produção Integrada começou com as frutas (PIF) ocorreu em 2001 e até agora mais de uma dezena de frutas já tiveram aprovadas e publicadas as suas normas técnicas específicas. 
Cada um dos procedimentos produtivos é agora encarado de forma sistêmica e não mais de forma isolada. Assim, ao invés da simples pulverização de agrotóxicos, propõe-se o combate às pragas e doenças através do manejo integrado de todos os meios de combate (biológicos, culturais e químicos), sendo que a utilização de defensivos químicos deve ser limitada ao mínimo, só sendo utilizada quando for absolutamente indispensável. Nesse método, privilegiam-se os meios indiretos de combate às pragas e doenças com práticas, sobretudo de caráter preventivo com a intenção de criar condições desfavoráveis para o seu desenvolvimento. Amaro (2003, p. 95 a 118) cita vários aspectos desse tipo de manejo que teria o mérito de fazer com que as plantas tenham melhores condições para resistir às pragas e infestantes.

Não se trata apenas de fornecer os nutrientes minerais disponíveis no solo, mas garantir que eles estejam disponíveis para as plantas e para isso é fundamental, por exemplo, a manutenção dos níveis adequados de atividade biológica.

Todos esses procedimentos devem estar aptos a se transformar em um banco de dados e informações para que se possa estabelecer um sistema de rastreabilidade total do processo produtivo. Dessa forma, torna-se possível uma avaliação dos procedimentos empreendidos pelo produtor em cada etapa do ciclo. $\mathrm{Na}$ medida em que ocorra algum problema com algum lote de produto, o sistema deve ser capaz de identificar exatamente onde e de que maneira esse problema teria ocorrido, possibilitando a sua correção.

\section{A PRODUÇÃo INTEGRADA NA PRÁTICA}

A metodologia do SAPI está sendo desenvolvida para identificar todos os passos e procedimentos culturais para orientar o produtor na sua adoção.

Dessa forma, em função de termos no território brasileiro diferentes características edafoclimáticas, não se pode definir a PI de uma planta para todo o território nacional, pois há que haver uma vinculação com a realidade específica e as características locais do desenvolvimento das culturas.

Para se chegar à formatação das denominadas notas técnicas específicas é necessário que se proceda a um processo de pesquisa e avaliação da cultura para a qual se quer estabelecer os princípios da PI em uma determinada região. Com a 
definição de todos os aspectos específicos da cultura em questão, os procedimentos culturais e de pós-colheita são sumarizados e devem estar, evidentemente, em consonância com os princípios gerais do SAPI.

A regulamentação específica da cultura é então instituída e se transforma em um manual de orientações para quem quer aderir ao sistema. Na prática, a definição de todos esses procedimentos significa um resgate do conhecimento secular dos produtores agrícolas, adicionado com os avanços técnicos da modernidade e submetidos à necessidade de proteção ambiental e da adoção de condições de trabalho minimamente aceitáveis.

Evidentemente se trata de uma mudança de paradigma produtivo que apresenta dificuldades na sua implantação, sobretudo por exigir procedimentos que foram soterrados pelo paradigma da "Revolução Verde". Mas ao padronizar as práticas produtivas teremos um controle específico de todo o processo produtivo.

A adesão ao SAPI acrescentaria mais alguns controles e procedimentos entre aqueles que já são exigidos pelas redes varejistas e, além disso, proporcionaria um reconhecimento da adoção de uma produção segura e sustentável através de certificação do sistema. Assim, o controle da segurança e qualidade dos produtos seria proporcionado a todos, do pequeno produtor ao produtor distribuidor.

Sem dúvida, isso tem um importante valor simbólico e justamente por isso um grande impacto comercial, sobretudo se houver adesão significativa dos produtores.

\section{UMA “REVOLUÇÃO DUPLAMENTE VERDE”?}

Muitos afirmam que seria necessária uma nova revolução verde, mas agora dirigida aos agricultores pobres e que tenha como objetivo a utilização dos solos de baixa qualidade, além de impactar menos o ambiente, ao contrário da primeira. "Uma revolução que seja ainda mais produtiva do que a primeira Revolução Verde e ainda mais verde em termos de conservação dos recursos naturais e do meio ambiente". (Conway, 2003, p. 68).

Há quem veja nessa tese apenas um conjunto de medidas de caráter paliativo baseado na "incorporação parcial de elementos de caráter ambientalista ou conservacionista nas práticas agrícolas convencionais (greening process), o que se constitui numa vã tentativa de recauchutagem do modelo da Revolução Verde". (Caporal e Costabeber, 2004, p. 8). 
Outros não questionam a proposta em si, mas argumentam que o processo é um pouco mais complexo pois não existiriam as condições de sua implantação e propagação de forma rápida.

Independente do nível em que se deem as objeções, considero fundamental um aspecto desse processo que é o fato dos produtores convencionais e as políticas públicas de diversos países passarem a incentivar métodos alternativos ao agroquímico, mesmo que combinados com ele. Dessa forma, novamente passa a não soar tão estranho para os agricultores a referência ao controle biológico de pragas e doenças, a preservação do material orgânico do solo e sua riqueza biológica, práticas comuns da antiga agricultura tradicional, mas agora potencializadas pelo avanço das pesquisas e das técnicas.

Pode ser talvez um passo importante para a incorporação dos princípios sustentáveis na agricultura num processo de transição que se coloca na perspectiva do longo prazo.

\section{CONSIDERAÇÕES FINAIS}

O processo de certificação imposto pelas grandes redes varejistas segue sendo implementado agora com algum tipo de negociação com algumas associações representantes dos produtores. A adesão ao processo de certificação é condição para o fornecimento para essas redes e os custos das auditorias e demais exigências têm recaído sobre os produtores. Entretanto, como afirmamos, os fornecedores desses produtores credenciados não são objeto de nenhuma dessas exigências e auditorias, o que coloca em cheque os objetivos reais desse processo. E vejam que o número desses fornecedores terceirizados não é insignificante: somente a Aphortesp (Associação dos Produtores e Distribuidores de Hortifruti do Estado de São Paulo) conta com 12 grandes produtores associados e credenciados que tem mais de 700 produtores parceiros e fornecedores que entregam diariamente seus produtos para 1.200 estabelecimentos comerciais e 250 cozinhas industriais.

Se a falha é evidente, qual o objetivo real então dessa política de credenciamento e controle?

Em relação às políticas públicas, existem atualmente no Brasil vários programas e sistemas voltados para obtenção de alimentos seguros que "foram implantados com a finalidade de disponibilizar, ao agronegócio, uma ferramenta a 
mais para fazer frente às exigências dos mercados, principalmente da Comunidade Europeia." (Kososki e Portocarrero, 2008, p. 20). Note-se que o objetivo declarado é o de responder a exigências do mercado exportador e realmente a maioria dos projetos de implementação da Produção Integrada estão relacionados com a produção de frutas especialmente para a exportação. Trata-se nesse caso, simplesmente de atender as exigências dos importadores.

Com base na experiência da produção integrada de frutas, o MAPA buscou expandir o projeto para outras cadeias produtivas agrícolas não necessariamente vinculadas ao setor exportador. Ok, a Produção Integrada permite que haja segurança na produção de alimentos, rastreabilidade e padronização dos processos produtivos mas é um programa de adesão voluntária.

Os produtores interessados em exportar são praticamente obrigados a aderir a esse programa se quiserem ter acesso ao mercado. A situação é diferente quando se trata do mercado interno onde o grande público simplesmente desconhece o que significa a "Produção Integrada", o que minimizaria as vantagens dos produtores que possam aderir ao sistema que, para ser implantado, necessita de um esforço gerencial e investimento em tecnologia e treinamento de mão de obra.

E mais, não se nota no conteúdo das políticas públicas dirigidas ao setor qualquer iniciativa séria de enfrentar decisivamente essa questão que então continua a depender da "mão invisível do mercado".

\section{REFERÊNCIAS}

ALTIERI, M. Agroecologia: a dinâmica produtiva da agricultura sustentável. Porto Alegre: UFRGS, 2004.

AMARO, P. Instituto Superior de Agronomia. www.isa.utl.pt, 2003. Acesso em: 15 jan. 2013.

ANDRIGUETO, J. R. Marco legal da produção integrada de frutas do Brasil, Brasília, 2002.

BERTALOT-BAY, M. M. Consequências ambientais e sociais da atividade agrícola, Campinas, 2008. Dissertação de mestrado.

BRUNDTLAND, G. H. Nosso Futuro Comum. Comissão Mundial sobre o Meio Ambiente e Desenvolvimento. Rio de Janeiro. 1988.

CARSON, R. Primavera Silenciosa. São Paulo: Melhoramentos, 1969. 
CONWAY, G. Produção de alimentos no século XXI. São Paulo: Estação Liberdade, 2003.

CORIAT, B. Pensar pelo avesso: o modelo japonês de trabalho e organização. Rio de Janeiro: Revan, 1994.

COSTABEBER, J. A.; CAPORAL, F. R. Agroecologia: alguns conceitos e princípios, Brasília, 2004.

KOSOSKI, A. R.; PORTOCARRERO, M. A. Alimentos seguros - uma política de governo. In: ANDRIGUETO, J. R., et al. Produção integrada no Brasi: agropecuária sustentável e alimentos. Brasília: Brasil. Ministério da Agricultura, Pecuária e Abastecimento, 2008. Cap. 1.

MAPA/IBAMA/ANVISA. Instrução Normativa Conjunta № 1. Brasília. 2010.

MAZOYER, M.; ROUDART, L. História das agriculturas no mundo: do neolítico à crise contemporânea. São Paulo: Unesp, 2010.

PEREIRA, D. Espacialidade da produção e do trabalho Industrial. São Paulo: PUCSP, 2001. Tese de Doutorado.

PEREIRA, D.; ABDO, R. Hortaliças e Rastreabilidade. Horticultura Brasileira, Brasília, v. 30, n. 2, 2012.

VEIGA, J. E. Desenvolvimento sustentável, alternativas e impasses. In: KEINERT, T. M. M. Organizações Sustentáveis: utopias e inovações. São Paulo: AnnaBlume, 2007. 
even under the natural appearance of limits, they are natural references (geographic forms), don't let pass as a socio historical translation.

We could also affirm that the reality here is the expression and the translation of who live in the studied territory, study that developed by collective methodologies that aimed to break, or even attenuate, the vertical relation that characterize the science in its dichotomy, between the research object and the subject. We worked to elaborate a study in which the construction/translation of 'reality' was not only produced by the researchers (dominant perspective), but like a conjunction of perspectives, represented by the researchers together with the people that participated as subject of their own process.

Our simple experience to produce science in a participative way, in our point of view, try to dialog with the Bruno Latour (2001) observations, principally breaking the knowledge perception funded into the dualism represented in the expressions: subject/object and watcher/watched. This duality should be thought as necessary to the science rationality, because should be as corollary for the whole translation of one reality. Contrarily, our work tried to break this duality, but even doing so, could we get the whole reality translation, or the closest from what we could call of truth?

Bruno Latour (2001) comments about the use of maps in scientific researches, and how the scientist dominate the world; however this same world has to be translated into concepts and forms: yes, the scientists dominate the world - but since the world came until them in the form of bidirectional inscriptions, super posts and combined. If this happens with the science production, we perceive that the same happen with the ethno maps, since the references that constitute the drawn and the map are social representations that the Pataxó people do of their territory, there are codes, concepts and inscriptions that mean the living world and its spatiality.

However we find out that the ethno map Pataxó produced (even elaborated from the Pataxó point of view), was favored by the knowledge and instruments elaborated from other disciplines areas represented on the use do GPS and GIS. So, the georeferenced positions are well accepted because the precision that the equipments are known to have, but even in face of this aspect of precision available in the market, they don't spoken about the world, but build representations that sometime are pushed far way, other time approached. In the same way occur with the Pataxó's representation about their territory, when they draw their community, 
their representation, theydon't talk about the own world, but about the world as a stage of a theater where those representation are realized.

\section{CONCLUSIONS}

In this sense, we also observed the difference of the maps made without the participation of its actors, which consists in a representation of certain space determined by the community as important and not the whole space or the space determined for someone else. The confection of the ethno maps and the discussion of them generated reflections regarding the local reality. Were thought situations from current time, that deviate of the old scenario, as change in the cultivation system, the growth number of the families, and the amount and water quality.

The reflections about the space, in turn, facilitated the perception of vulnerability factors and of measures that are, or are not being taken to regarding them. In that sense, was possible to meditate as expected, the cultural, social, political and environmental aspects for the social technology construction.

In this context the digital technology is indispensable and the resources, limited. Still, the used resources were understood by the community that participated in the whole process. However, although it is still restricted the access to instruments (GPS) and to computer programs for mapping, the activity is viable for indigenous communities. The indigenous, like most Brazilians, have easiness using cell phones or devices like music and video players, that don't differ a lot in commands and handling, such like a standard GPS.

We noticed that is possible to promote the exercise of political power by local traditional people, to lead their cultural diversity and biodiversity conservation. It is important to point out that the community development in this process can be stimulated by the construction of autonomy and empowerment of those traditional people.

\section{Acknowledgements}

To the all Imbiruçu indigenous for the support in this study. Especially to the Cacique Romildo Pataxó and the Pataxó students Keyla Francis de Jesus da Conceição and Wakey Silva dos Santos (Junior Scientific Initiation Scholarship, UFVJM). To the Fundação de Amparo a Pesquisa do Estado de Minas Gerais (Fapemig) and to the National Council for Scientific and Technological Development (CNPq), for the scholarships. 


\section{REFERENCES}

ANGTHICHAY, ARARIBY, JASSANÃ, MANGUAHÃ, \& KANÁTYO. O povo Pataxó e suas histórias. 6.ed. São Paulo: Global, 47p., 2002.

BLACK, J. Mapas e história: construindo imagens do passado. 1.ed. Bauru, SP: EDUSC, 428p., 2005.

CAMPOS, R.C. Movimentos indígenas por educação: novos sujeitos socioculturais na história recente do Brasil. In: Reunião Anual da ANPED, n.23, Caxambu, 2000.

CARVALHO, A. $\mathbf{O}$ geoprocessamento na gestão ambiental em Terras Indígenas: uma experiência com etnomapeamento junto à Comissão Pró-Índio do Acre. Science Master Dissertation (Physical Geography). Geography Department, Faculdade de Filosofia, Letras e Ciências Humanas, USP, São Paulo, 127p., 2006.

CEDEFES. Centro de Documentação Eloy Ferreira da Silva. A luta dos índios pela terra: Contribuição a história indígena de Minas Gerais. 1.ed. Contagem, 120p., 1987.

CEDEFES. Centro de Documentação Eloy Ferreira da Silva. (2006). Pataxó: Quem é esse povo? Disponível em: $<$ http://www.cedefes.org.br/new/index.php?conteudo=materias/index\&secao=1\&tema $=53 \&$ materia $=1774>$ Acesso em: 15/06/2010.

CHAMBERS, R. The origins and practice of participatory rural appraisal. World Development, v. 22, n. 7, p. 953-969, 1994.

CHAVES, A. S.; COLLI, F. Processos educativos Revista Terra Viva: estratégias, ações, resultados e desafios na disseminação agroflorestal. Brasília: Ministério do Meio Ambiente. Série Sistematização v. 1, p. 22-33, 2006.

CORREIA, C. S. Etnozoneamento, etnomapeamento e diagnóstico etno ambiental: representações cartográficas e gestão territorial em Terras Indígenas no Estado do Acre. PhD Thesis (Antropology) - Antropology Department, UnB, Brasília, 420p., 2007.

FARIA, A. A. C. O uso do diagnóstico rural participativo em processos de desenvolvimento local: um estudo de caso. Dissertation (Master Science) Universidade Federal de Viçosa, UFV, Viçosa, 111p., 2000.

FREIRE, P. Pedagogia da autonomia - Saberes necessários à prática educativa. São Paulo: Paz e Terra, 37 ed., 148p., 1996.

HARLEY, J. B. Maps, knowledge, and power. In: Cosgrove, Denis \& Daniels, Stephen (org.). The iconography of landscape: Essays on the symbolic representation, design and use of post environments. Cambridge University Press, 1 ed., 162p, 1988. 
HERLIHY, P. H.; KNAPP, G. Maps of, by, and for the peoples of Latin America. In: Human organization. Journal of the Society for Applied Anthropology, v. 62, n. 4, p. 303-314, 2003.

IBGE. Instituto Brasileiro de Geografia e Estatística. (2007). <http://www.ibge.gov.br> LATOUR, B. A esperança de Pandorra: Ensaios sobre a realidade dos estudos científicos. Bauru, SP : EDUSC, 2001, 372p.

LAURIOLA, V. Ecologia global contra diversidade cultural? Conservação da natureza e povos indígenas no Brasil. O Monte Roraima entre Parque Nacional e a Terra Indígena Raposa-Serra do Sol. Revista Ambiente \& Sociedade, v. 5, n. 2, p. 165189, 2003.

LITTLE, P. Gestão territorial em Terras Indígenas: definição de conceitos e proposta de diretrizes. Secretaria de Estado de Meio Ambiente e Recursos Naturais - SEMA-AC, Secretaria Extraordinária dos Povos Indígenas - SEPI-AC e Agência de Cooperação Técnica Alemã (GTZ) no Brasil - GTZ, Rio Branco, Acre, 2006.

MELO, W. F., SILVA, S. S., TAVARES, R. A., FRESCHI, J. M., GAVAZZI, R. A., SILVA, J. F. M., PIYANKO, B., APIWTXA, C. A., BROWN, I. F. Aplicação de dados SRTM, sensoriamento remoto e SIG em etnomapeamento: o caso da Terra Indígena Kampa do Rio Amônia na fronteira Brasil-Acre/Peru-Ucayali. In: Simpósio Brasileiro de Sensoriamento Remoto, INPE, n.13, Florianópolis, SC, 2007. Disponível em: <http://marte.dpi.inpe.br/col/dpi.inpe.br/sbsr@80/2006/11.15.23.05/doc/59495956.pdf $>$ Acesso em 15/06/2010

ORLOVE, B. Mapping reeds and reading maps: the politics of representation in Lake Titicac. American Ethnologist, v. 18, n. 1, p. 3ᄀ38, 1991.

PATAXÓ, R. Encontro com o povo Pataxó de Carmesia. Grupo de Estudos dos Povos Indígenas de Minas Gerais. Universidade Federal dos Vales do Jequitinhonha e Mucuri (UFVJM). Campus I Diamantina, Brazil, 2008.

SCHWARTZMAN, S., ZIMMERMAN, B. Conservation alliances with indigenous peoples of the Amazon. Conservation Biology, v. 19, n. 3, p. 721-727, 2005.

SMITH, D. A. Participatory mapping of community lands and hunting yields among the bugle of Western Panama. Human Organization. Journal of the Society for Applied Anthropology, v. 62, n. 4, p. 332-343, 2003.

THE NATURE CONSERVATION. Etnomapeamento nas comunidades indígenas

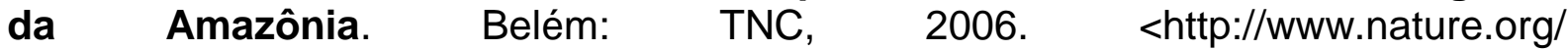
wherewework/southamerica/brasil/work/art16607.html>

VERDEJO M. E. Diagnóstico Rural Participativo. Guia Prático. 1.ed. Brasília: Ministério do Desenvolvimento Agrário, 62 p., 2006. 
VIRGILIO H, BARROS M. V. Z SIG aplicado à caracterização sócio ambiental da reserva indígena Apucarana (PR). Estudos Geográficos, v. 5, n. 1, p. 47-62, 2007. Disponível em: <http://cecemca.rc.unesp.br/ojs/index.php/estgeo>. Acesso em 15/06/2010. 